\title{
QUALIDADE DE VIDA DE IDOSOS COM INSUFICIÊNCIA CARDÍACA
}

\author{
QUALITY OF LIFE OF ELDERLY PEOPLE WITH HEART FAILURE
}

\section{CALIDAD DE VIDA DE PERSONAS MAYORES CON INSUFICIENCIA CARDÍACA}

\author{
Mariana Manhezi Bonifacio De Sousa Silva* \\ Claudia Silva Marinho** \\ Elieusa Silva Sampaio*** \\ Rosana Dos Santos Silva**** \\ Cláudia Geovana Da Silva Pires***** \\ Éverton Nascimento Fraga ${ }^{* * * * * *}$
}

\section{RESUMO}

Objetivo: Avaliar a qualidade de vida de idosos com diagnóstico de insuficiência cardíaca acompanhados no ambulatório de um hospital universitário em Salvador, Estado da Bahia, Brasil. Material e Método: Estudo avaliativo, realizado com 68 idosos portadores de insuficiência cardíaca. Os dados foram coletados a partir das escalas WHOQOL-OLD e BREF, e analisados por meio da estatística descritiva. Resultados: Houve equivalência entre os sexos dos participantes, a maioria (64,7\%) tinha entre 60 e 69 anos, 86,8\% aposentados, $83,8 \%$ ensino fundamental, $92,6 \%$ renda de 1 a 3 salários mínimos, 82,3\% moravam com 1 ou mais pessoas e 72,1\% eram provedores exclusivos do lar. Os fatores relacionados aos domínios Físico $(83,8 \%)$ e Meio Ambiente (86,8\%) influenciaram para uma pior qualidade de vida (média 1,96 e 1,76 de 5 respectivamente), e os relacionados ao Funcionamento do Sensório (51,5\%) e Morte e Morrer (47,1\%) à melhor qualidade de vida (média 2,43 e 2,28 de 5 respectivamente). Conclusão: A qualidade de vida desses idosos está aquém do ideal. A manutenção dos impactos das doenças cardíacas e o incremento no envelhecimento em âmbito global torna essencial o conhecimento proporcionado pelo estudo para desenvolvimento de estratégias de controle da doença, melhoramento da qualidade de vida e redução de gastos com a saúde.

*Enfermeira, Especialista em Saúde do Adulto com Ênfase Cardiovascular, Hospital Universitário Professor Edgard Santos, Salvador, Bahia, Brasil, ORCID: https://orcid.org/0000-0001-8938-794 Email: mari_manhezi@hotmail.com Autora correspondente.

**Enfermeira, Dra. em Ciências, Escola de Enfermagem da Universidade Federal da Bahia, Salvador, Bahia, Brasil, ORCID: https://orcid.org/0000-0002-0597-8126 Email: marinho-claudia@hotmail.com Autora correspondente.

***Enfermeira, Dra. em Medicina e Saúde, Escola de Enfermagem da Universidade Federal da Bahia, Salvador, Bahia, Brasil, ORCID: https://orcid.org/0000-0003-1702-7296 Email: eesampaio@ufba.br.

****Psicóloga, Dra. em Ciências Sociais, Hospital Universitário Professor Edgard Santos, Salvador, Bahia, Brasil, ORCID: https:// orcid.org/0000-0003-4830-6781 Email: rosanassilva@hotmail.com

${ }^{* * * * *}$ Enfermeira. Dra. em Enfermagem, Escola de Enfermagem da Universidade Federal da Bahia, Salvador, Bahia, Brasil, ORCID: https://orcid.org/0000-0001-9309-2810 Email: cgspires@ufba.br.

******Enfermeiro. Escola de Enfermagem da Universidade Federal da Bahia, Salvador, Bahia, Brasil, ORCID: https://orcid. org/0000-0002-1251-9868 Email: everton_365@live.com. 
Palavras-chave: Insuficiência cardíaca; Idosos; Serviços de saúde para idosos; Qualidade de vida; Geriatria; Inquéritos e questionários.

\begin{abstract}
Objective: To analyze the quality of life of elderly patients diagnosed with heart failure in an outpatient clinic of a university hospital in Salvador, State of Bahia, Brazil. Material and Method: Evaluative study performed with 68 patients with heart failure. Data was collected from WHOQOL-OLD and BREF scales and then analyzed using descriptive statistics. Results: There was similar gender representation. Most of the participants were between 60 to 69 years old $(64.7 \%)$, retired (86.8\%), had attended primary school $(83.8 \%)$, received 1 to 3 minimum wages $(92.6 \%)$, lived with 1 or more people $(82.3 \%)$ and $72.1 \%$ were the sole providers of their families. The elements related to the Physical (83.8\%) and Environmental Domain (86.8\%) were linked to a worse quality of life (average 1.96 and 1.76 of 5, respectively) and those related to Sensory Functioning (51.5\%) and Death and Dying (47.1\%) were linked to a better quality of life (average 2.43 and 2.28 of 5, respectively). Conclusion: The quality of life of the assessed population is worse than expected. The impact from heart disease and the increase in aging globally makes this study essential for the development of strategies to control the disease, improve the quality of life and reduce healthcare costs.
\end{abstract}

Key words: Heart failure; Elderly; Health Services for the Elderly; Quality of life; Geriatrics; Surveys and Questionnaires.

\title{
RESUMEN
}

Objetivo: Evaluar la calidad de vida de las personas mayores diagnosticadas con insuficiencia cardíaca y en seguimiento en la clínica ambulatoria de un hospital universitario en Salvador, Estado de Bahía, Brasil. Material y Método: Estudio evaluativo, realizado con 68 personas mayores con insuficiencia cardíaca. Los datos fueron recolectados usando las escalas WHOQOL-OLD y BREF, y analizados con estadística descriptiva. Resultados: Hubo equivalencia entre los sexos de los participantes, la mayoría (64, 7\%) tenía entre 60 y 69 años, 86, 8\% jubilados, $83,8 \%$ con educación primaria, 92,6\% ingresos de 1 a 3 salarios mínimos, 82,3\% vivían con 1 o más personas y $72,1 \%$ eran proveedores exclusivos del hogar. Los factores relacionados con los dominios Físico (83,8\%) y Medio Ambiente (86,8\%) influyeron en una peor calidad de vida (promedio 1,96 y 1,76 de 5 respectivamente), y aquellos relacionados con la Función Sensorial $(51,5 \%)$ y Muerte y morir $(47,1 \%)$ para la mejor calidad de vida (promedio 2,43 y 2,28 de 5 respectivamente). Conclusión: La calidad de vida de estas personas mayores es menos que ideal. Mantener los impactos de las enfermedades cardíacas y aumentar el envejecimiento global hace que el conocimiento proporcionado por el estudio sea esencial para desarrollar estrategias para controlar la enfermedad, mejorar la calidad de vida y reducir los costos de salud.

Palabras clave: Insuficiencia cardíaca; Personas mayores; Servicios de salud para ancianos; Calidad de vida; Geriatría; Encuestas y cuestionarios.

Data de recepção: 25/08/2020

Data de aceitação: 17/01/2021

\section{INTRODUÇÃO}

A insuficiência cardíaca (IC) é uma complicação prevalente na maioria dos indivíduos cardiopatas, se tornando um problema de saúde pública importante na atualidade. É a principal causa de internação por doenças cardiovasculares, sendo responsável por um índice elevado de morbimortalidade, ocasionando altos custos financeiros ao Sistema Único de Saúde Brasileiro (SUS). Assim, faz-se necessário um atendimento integral, com abordagem multiprofissional, visando a redução das suas complicações e as admissôes e readmissões hospitalares ${ }^{(1)}$. 
A maioria dos pacientes que foram acometidos pela IC no Brasil, no período de 2011 a 2012, foi de idosos, principalmente nas regióes sul e sudeste. A etiologia isquêmica teve maior predominância, sendo a má adesão medicamentosa o principal fator para descompensação da doença ${ }^{(2)}$. Este estudo demonstrou que a prevalência de pessoas maiores de 18 anos com IC tenderá a aumentar até 2030, resultando em mais de 8 milhóes de portadores, sendo a principal causa atribuída a este fato, o aumento da expectativa de vida, uma vez que a IC é mais prevalente nas faixas etárias mais elevadas ${ }^{(3)}$.

Diante desse contexto, o Brasil enfrenta crescentes demandas de sua população que envelhece, promovendo políticas públicas que proporcionem um envelhecimento com qualidade de vida. Esse fato revela a importância de estudos que subsidiem estratégias que resultem em melhores condiçôes de saúde da população idosa ${ }^{(4)}$.

A saúde dos indivíduos idosos com doenças cardiovasculares é prejudicada por fatores físicos e psíquicos, resultando num comprometimento da qualidade de vida. A presença de aspectos limitantes da saúde, como a capacidade funcional diminuída e problemas emocionais, estão presentes na rotina desses indivíduos, repercutindo nas realizaçóes de atividades de vida diária e na vida social ${ }^{(5)}$.

Estudos alusivos ao conhecimento da qualidade de vida (QV) têm possibilitado o entendimento a respeito da natureza e extensão dos problemas funcionais e biopsicossociais durante a trajetória da doença, especialmente das doenças crônicas. Dessa forma, o tratamento adequado pode ser proposto pelo profissional de saúde com maior segurança ao portador da doença, visando uma melhor assistência e reabilitação precoce ${ }^{(6)}$.

A QV relacionada à saúde é uma medida multidimensional e subjetiva. Pode ser influenciada por fatores socioeconômicos, demográficos, relacionados às condiçóes de saúde e estilo de vida do indivíduo, sendo útil para guiar açóes de cuidado integral e promoção da saúde ${ }^{(7)}$.

$\mathrm{O}$ conceito de QV é amplo, com múltiplas definições na literatura, e depende da influência de aspectos e percepçóes individuais, ambientais e sociais. A análise da situação de saúde e QV de uma população permite estabelecer o perfil da mesma e traçar metas de intervenção de acordo com as necessidades existentes ${ }^{(8)}$.

A partir do conhecimento das características dos grupos de indivíduos acometidos por IC e os principais fatores que interferem na sua $\mathrm{QV}$, é possível direcionar e fortalecer a prática da Enfermagem nesse âmbito, visando o melhor atendimento integral no controle das doenças, e, consequentemente, melhores condições de saúde para esses indivíduos e aperfeiçoamento do cuidado prestado $^{(4)}$.

Os indivíduos idosos são naturalmente afetados por perdas e carências, e quando somado com a presença de doenças, incapacidade/dependência e solidão, observa-se uma piora da QV nessa população. A presença de apoio social e familiar nessa etapa da vida contribuem para uma melhor QV e bem estar ${ }^{(9)}$.

Além disso, no contexto brasileiro, os ambulatórios especializados são gerenciados por enfermeiras, e este estudo pode fornecer maior entendimento das características e necessidades pessoais dos idosos com IC e subsidiar estratégias de cuidados integrais a esses indivíduos, com redução de complicaçóes na sua saúde, internações hospitalares e custos ao sistema de saúde.

Para tanto, o objetivo geral do estudo é avaliar a qualidade de vida de idosos com diagnóstico de insuficiência cardíaca acompanhados no ambulatório de um hospital universitário em Salvador, Bahia.

\section{MATERIAL E MÉTODO}

Trata-se de uma pesquisa de campo, prospectivo, de caráter avaliativo com abordagem quantitativa, realizada com idosos com diagnóstico de IC acompanhados em ambulatório de referência em cardiologia de um hospital em Salvador, Bahia.

Dentre a população, a amostra foi constituída por conveniência, onde os idosos foram selecionados por meio dos seguintes critérios de inclusão: ter 60 anos ou mais de idade, confirmação diagnóstica de IC (crônica); ter condiçôes físicas, mentais e intelectuais para responder o questionário; e aceitar livremente participar da pesquisa.

A coleta de dados foi realizada através da aplicaçáo de dois instrumentos de coleta: um questionário para obter dados que dizem respeito à caracterização sociodemográfica dos pacientes $\mathrm{e}$ a escala de QV já validada, amplamente utilizada na literatura científica sobre o tema, WHOQOL- 
$\mathrm{OLD}^{(10)}$. A coleta de dados ocorreu durante um período de 3 meses (de 27 de julho a 26 de outubro de 2018).

O questionário para levantamento de características sociodemográficas, desenvolvido para a pesquisa, foi constituído de informaçóes sobre sexo, idade, raça/cor, profissão, ocupação, renda, condições de moradia, escolaridade, estado civil, religiăo e cidade de origem.

A escala WHOQOL-OLD foi desenvolvida por um grupo de especialistas em QV da Organização Mundial da Saúde ${ }^{(10)}$, com o intuito de criar um questionário direcionado a idosos levando em consideração as especificidades do fenômeno do envelhecimento. Dezoito países, dentre os quais o Brasil, foram responsáveis pela elaboração desse instrumento que possui 24 questóes, divididas em seis facetas, cada uma com quatro itens: Faceta 1: Funcionamento dos sentidos (FS), avaliando o funcionamento sensorial e o impacto da perda das habilidades sensoriais na QV; Faceta 2: Autonomia (AUT), sugestiva à independência na velhice; Faceta 3: Atividades passadas, presentes e futuras (PPF), descrevendo a satisfação sobre conquistas na vida e anseios; Faceta 4: Participação social (PSO), que apresenta a participação em atividades do cotidiano; Faceta 5: Morte e morrer (MEM), a qual se inclui as preocupaçóes, inquietaçóes e temores sobre a morte e morrer; e, Faceta 6: Intimidade (INT), que estima a capacidade de se ter relaçóes pessoais e íntimas $^{(11)}$.

Esta escala é utilizada em conjunto com o instrumento WHOQOL-BREF, também criada pela $\mathrm{OMS}^{(12)}$, pois se refere a uma avaliação complementar da QV. Esse contém 26 questóes, sendo duas gerais (QV geral e satisfação com a saúde) e 24 reunidas nos domínios físico, psicológico, relaçóes sociais e meio ambiente ${ }^{(13)}$.

Em ambos instrumentos são apresentadas 5 alternativas em todas as questóes, representadas pelos números de 1 a 5 . Ao final, as repostas são somadas e agrupadas por facetas (WHOQOLOLD) e domínios (WHOQOL-BREF), de forma que quanto mais próximas de 5, melhor a $\mathrm{QV}$, e quanto mais próxima de 1 , pior a $\mathrm{QV}^{(11)}$.

Os instrumentos foram aplicados pela pesquisadora e dois graduandos de enfermagem da Universidade Federal da Bahia, previamente treinados, aos pacientes antes ou após a consulta médica ambulatorial de rotina, de acordo com a preferência do entrevistado e a dinâmica do serviço, visando o conforto e garantindo a não interrupção durante o preenchimento dos dados.

Foi realizada a explicação prévia do instrumento do projeto de pesquisa, e os pacientes que aceitaram participar assinaram o Termo de Consentimento Livre e Esclarecido (TCLE), daí eram encaminhados a uma sala reservada para preenchimento dos instrumentos sem interrupçóes ou influências dos pesquisadores, no entanto, estes permaneceram sempre próximos e acessíveis para esclarecimentos.

Os dados foram digitados e analisados no Software estatístico IBM SPSS Statistics 14. A estatística descritiva foi utilizada para caracterização dos participantes e seus resultados apresentados em números absolutos e percentual simples. Os dados referentes à QV dos idosos foram apresentados por meio de média, mediana, desvio padrão $(\mathrm{dp})$ e variação.

As análises foram geradas a partir das respostas das 12 facetas/domínios que compóem as escalas. Cada resultado encontrado foi agrupado e analisado conforme a seguinte classificação de QV: 1) necessita melhorar (média de 1 até 2,9); 2) regular (média de 3 até 3,9); 3) boa (média de 4 até 4,9) e 4) muito boa (média de 5) ${ }^{(14)}$.

Esta pesquisa foi aprovada pelo Comitê de Ética e Pesquisa (CEP) do Hospital Universitário sob parecer $n^{\circ} 89102418.5 .0000 .0049$ e obedeceu aos princípios éticos que regem às pesquisas em saúde com seres humanos no Brasil ${ }^{(15)}$.

\section{RESULTADOS}

No total participaram do estudo 68 idosos por atenderem aos critérios de inclusão definidos. O perfil sociodemográfico desses participantes é demonstrado na Tabela 1.

É possível observar que houve uma equivalência entre o sexo dos participantes, dois homens a mais que mulheres, a maioria com idade entre 60 e 69 anos, raça/cor autodeclarada parda e residente na capital. A maioria dos idosos eram casados, possuíam ensino Fundamental, eram aposentados, com renda entre 1 a 3 salários mínimos (U\$246.19 a 738.58), residiam com mais de um membro familiar, e eram provedores exclusivos do lar.

A Tabela 2 apresenta a distribuição descritiva das facetas e domínios conforme as 4 classificações 
de QV, mostrando os números e frequência das respostas nas classificaçôes 1 e 2 (precisa melhorar respostas dos participantes. É possível notar maior e regular), respectivamente. Já os domínios predominância da classificaçâo QV 2 e menor da Funcionamento do Sensório e Morte e Morrer QV 4 dentre os domínios, evidenciando, no geral, alcançaram melhores pontuaçóes de QV, com uma QV declarada regular pelos participantes. Os domínios Físico e Meio Ambiente obtiveram menores pontuaçóes de $\mathrm{QV}$, nos quais $83,8 \%$ $51,5 \%$ e $47,1 \%$ dos participantes, concentrando suas respostas nas classificações 3 e 4 (boa e muito boa), respectivamente.

e $86,8 \%$ dos participantes concentraram suas

Tabela 1. Características sóciodemográficas de idosos acompanhados no ambulatório de Insuficiência Cardíaca de um Hospital em Salvador, Estado da Bahía, Brasil, 2018 (n= 68).

\begin{tabular}{|c|c|c|c|}
\hline Variáveis & & $\mathrm{n}(68)$ & $\%(100)$ \\
\hline \multirow[t]{2}{*}{ Faixa Etária } & 60-69 anos & 44 & 64,70 \\
\hline & $70-80$ anos & 24 & 35,30 \\
\hline \multirow[t]{2}{*}{ Sexo } & Masculino & 35 & 51,50 \\
\hline & Feminino & 33 & 48,50 \\
\hline \multirow[t]{3}{*}{ Raça/Cor } & Pardo & 39 & 57,35 \\
\hline & Preto & 22 & 32,35 \\
\hline & Branco & 7 & 10,30 \\
\hline \multirow[t]{2}{*}{ Profissão } & Sim & 40 & 58,80 \\
\hline & Não & 28 & 41,20 \\
\hline \multirow[t]{2}{*}{ Aposentado } & Sim & 59 & 86,80 \\
\hline & Não & 9 & 13,20 \\
\hline \multirow[t]{2}{*}{ Renda* } & $<1$ salário mínimo & 5 & 7,35 \\
\hline & 1 a 3 salários mínimos & 63 & 92,65 \\
\hline \multirow[t]{2}{*}{ Provedor do lar } & Sim & 49 & 72,06 \\
\hline & Não & 19 & 27,94 \\
\hline \multirow[t]{3}{*}{ Mora com você } & Ninguém & 12 & 17,60 \\
\hline & 1 pessoa & 26 & 38,20 \\
\hline & $>1$ pessoa & 30 & 44,10 \\
\hline \multirow[t]{3}{*}{ Escolaridade } & Fundamental & 57 & 83,80 \\
\hline & Médio & 11 & 16,20 \\
\hline & Superior & 0 & 0,00 \\
\hline \multirow[t]{3}{*}{ Estado civil } & Solteiro & 17 & 25,00 \\
\hline & Casado & 38 & 55,90 \\
\hline & Viúvo & 13 & 19,10 \\
\hline \multirow[t]{2}{*}{ Cidade de Origem } & Capital & 46 & 67,65 \\
\hline & Interior & 22 & 32,35 \\
\hline \multirow[t]{3}{*}{ Religião } & Católico & 46 & 67,60 \\
\hline & Evangélico & 11 & 16,20 \\
\hline & Outros $\dagger$ & 11 & 16,20 \\
\hline
\end{tabular}

*Considerando o valor: R\$ 954,00 (U\$ 246.19) como salário mínimo em 2018 (Decreto no 9.255 de 29/12/2017). †Outros: Espírita, Testemunha de Jeová, Agnóstico. 
Tabela 2. Distribuição das respostas em WHOQOL-OLD e WHOQOL-BREF dos idosos acompanhados no ambulatório de Insuficiência Cardíaca de um Hospital em Salvador, Estado da Bahia, Brasil, 2018 (n= 68).

\begin{tabular}{lcccc}
\hline Facetas/Domínios & $\mathbf{1}$ (precisa melhorar) & $\begin{array}{c}\mathbf{2} \text { (regular) } \\
\mathrm{n}(\%)\end{array}$ & $\begin{array}{c}\mathbf{3} \text { (boa) } \\
\mathrm{n}(\%)\end{array}$ & $\begin{array}{c}\text { 4 (muito boa) } \\
\mathrm{n}(\%)\end{array}$ \\
\hline WHOQOL-BREF & & & & \\
Qualidade de vida (geral) & $5(7,4)$ & $31(45,6)$ & $26(38,2)$ & $6(8,8)$ \\
Satisfação com saúde & $16(23,5)$ & $34(50,0)$ & $11(16,2)$ & $7(10,3)$ \\
Domínio físico & $14(20,6)$ & $43(63,2)$ & $11(16,2)$ & - \\
Domínio psicológico & $4(5,9)$ & $36(52,9)$ & $26(38,2)$ & $2(2,9)$ \\
Relaçóes sociais & $9(13,2)$ & $32(47,1)$ & $24(35,3)$ & $3(4,4)$ \\
Meio ambiente & $25(36,8)$ & $34(50,0)$ & $9(13,2)$ & - \\
\hline WHOQOL-OLD & & & & \\
Funcionamento sensório & $10(14,7)$ & $23(33,8)$ & $31(45,6)$ & $4(5,9)$ \\
Autonomia & $6(8,8)$ & $40(58,8)$ & $20(29,4)$ & $2(2,9)$ \\
Atividades passadas, pre- & $7(10,3)$ & $37(54,4)$ & $23(33,8)$ & $1(1,5)$ \\
sentes e futuras & & & & \\
Participação social & $11(16,2)$ & $37(54,4)$ & $19(27,9)$ & $1(1,5)$ \\
Morte e morrer & $18(26,5)$ & $18(26,5)$ & $27(39,7)$ & $5(7,4)$ \\
Intimidade & $24(35,3)$ & $17(25,0)$ & $22(32,4)$ & $5(7,4)$ \\
\hline
\end{tabular}

$\mathrm{Na}$ Tabela 3 observa-se que as médias entre os domínios variaram de 1,76 (Meio Ambiente) até 2,49 (Qualidade de vida). Os menores valores, indicando uma pior QV, são referentes aos domínios Físico e Meio Ambiente, que, inclusive, foram os únicos domínios que não apresentaram a classificação máxima de QV 4 (muito boa média de 5). Enquanto os maiores valores foram Qualidade de Vida e Funcionamento do Sensório.

Tabela 3. Respostas por média, mediana e variação em WHOQOL-OLD e WHOQOL-BREF dos idosos acompanhados no ambulatório de Insuficiência Cardíaca de um Hospital em Salvador, Estado da Bahia, Brasil. 2018 $(n=68)$.

\begin{tabular}{lccc}
\hline Facetas/Domínios & Média (dp) & Mediana & $\begin{array}{c}\text { Variaçáo } \\
\text { observada QV }\end{array}$ \\
\hline WHOQOL-OLD & $2,43(0,816)$ & 3,00 & $1-4$ \\
Funcionamento sensório & $2,26(0,661)$ & 2,00 & $1-4$ \\
Autonomia & $2,26(0,661)$ & 2,00 & $1-4$ \\
Atividades passadas, presentes e futuras & $2,15(0,697)$ & 2,00 & $1-4$ \\
Participação social & $2,28(0,944)$ & 2,00 & $1-4$ \\
Morte e morrer & $2,12(0,985)$ & 2,00 & $1-4$ \\
Intimidade & & & $1-4$ \\
\hline WHOQOL-BREF & $2,49(0,763)$ & 2,00 & $1-4$ \\
Qualidade de vida & $2,13(0,896)$ & 2,00 & $1-3$ \\
Satisfação com saúde & $1,96(0,609)$ & 2,00 & $1-4$ \\
Domínio físico & $2,38(0,647)$ & 2,00 & $1-4$ \\
Domínio psicológico & $2,31(0,758)$ & 2,00 & $1-3$ \\
Relaçóes sociais & $1,76(0,672)$ & 2,00 & \\
Meio ambiente & &
\end{tabular}




\section{DISCUSSÃO}

Os resultados, quanto a faixa etária e sexo, corroboram com outros estudos brasileiros que evidenciaram numa população de pacientes internados com IC, que as médias das idades prevalentes foram de 65 e 66 anos, respectivamente, sendo a maioria do sexo masculino ${ }^{(16,17)}$. Também, estudo que analisou as internaçóes por IC no Brasil, no período de 2001 a 2012, constatou ser brasileiros homens e idosos que mais internam por $\mathrm{IC}^{(18)}$.

Ainda, os dados dessa pesquisa confirmam os resultados presentes em outro estudo ${ }^{(19)}$, que aponta que, no geral, a pessoa idosa, independente da condição social e de saúde, ainda mantém a condição de mantenedora da família, possuindo duas ou três pessoas dependentes da sua renda. A autora explica que esse fato pode estar associado à dificuldade atual do jovem se inserir no mercado de trabalho, no aumento do desemprego e falta de políticas de inclusão, resultando na permanência desse indivíduo por mais tempo no lar de origem, tendo o idoso como provedor principal.

A aposentadoria dos idosos corresponde a uma parte importante na renda das famílias em que estão inseridos, contribuindo para o surgimento de uma nova função da populaçấo envelhecida na sociedade atual, a de provedor ou pilar econômico da família ${ }^{(20)}$.

Ainda, segundo a Pesquisa Nacional por Amostra de Domicílios - PNAD ${ }^{(21)}$, no nordeste brasileiro, as pessoas que mais foram apontadas como "pessoa de referência" no domicílio, tinham idade de 60 anos ou mais, com tempo de estudo entre 4 e 14 anos, e renda variante de mais de meio até dois salários mínimos.

Quanto à religião, 67,6\% dos idosos se declararam católicos, corroborando com outro estudo em que essa porcentagem equivale a $69,5 \%$. Os autores afirmam que o relacionamento com Deus é maior com o aumento da idade, resultando em um desenvolvimento espiritual positivo, e acarretando em um envelhecimento saudável; ainda destacam que a espiritualidade e religiosidade proporcionam maior sentido de vida, contribuem para o suporte social e emocional, além da melhoria da saúde e $\mathrm{QV}^{(22)}$.

Nos instrumentos direcionados a QV, as perguntas "Você tem dinheiro suficiente para satisfazer suas necessidades?" e "Em que medida você tem oportunidades de atividade de lazer?", foram as que obtiveram menores médias como respostas no domínio Meio Ambiente (1,76; dp: 0,672 ), indicando a influência negativa da restrição financeira e falta de oportunidade de lazer para a QV desses idosos. Estudo brasileiro, em região semelhante, com 113 idosos, que aborda QV no contexto do trabalho, evidenciou que escores médios mais baixos estão ligados a esse domínio, e apesar de não ter encontrado relação estatística ( $\mathrm{p}=$ $0,630)$, infere que esse fato pode estar relacionado a baixa escolaridade evidenciada nos idosos (61,9\% nível fundamental incompleto), assim como neste estudo $(83,8 \%)$, haja visto que indivíduos com reduzido nível de instrução têm dificuldades na aquisição de novas informaçóes, no acesso e na realização de cuidados adequados a manutenção da saúde ou prevenção de agravo e doenças ${ }^{(23)}$.

A atividade de lazer durante a terceira idade possibilita uma ressignificação, na perspectiva do próprio individuo, do que é essa fase da vida. Os aspectos negativos do envelhecimento são afastados nesse processo, de modo que sentimentos como solidão e perda sejam descartados, e novas conquistas, relaçóes e aprendizados sejam construídos e agregados. Uma atividade de lazer reafirma a capacidade da pessoa idosa de manter e negociar identidades, bem como assinalam mudanças importantes de atitudes, hábitos e comportamentos $^{(24)}$.

Poder econômico, lazer e QV estão relacionados em uma pesquisa com idosos acerca da representação social dessa população, evidenciado que o dinheiro proporciona a esses indivíduos uma alimentaçâo de qualidade e prática de atividades de lazer, estando esta última relacionada à satisfação social $^{(4)}$. A vivência do lazer é influenciada pela presença e as formas de uso do espaço público, dependendo da atuação de grupos políticos, religiosos e culturais, bem como a proximidade ou distância de determinadas áreas da cidade. $\mathrm{O}$ acesso democrático a serviços e equipamentos culturais ocorre com maior abrangência nas zonas centrais das cidades brasileiras, que são econômica e espacialmente segregadas. Os habitantes de bairros populares convivem com limitaçóes de mobilidade, além de barreiras geográficas e financeiras ${ }^{(25)}$. Este fato se torna mais agravante quando é realidade de pessoas idosas, dificultando ainda mais a prática de lazer. 
A segunda menor média foi no domínio Físico (1,96; dp: 0,609), as perguntas com menores escores foram "Em que medida você acha que sua dor (física) impede você de fazer o que você precisa?" e "Você tem energia suficiente para seu dia-a-dia?". Os dados corroboram com o achado de outro estudo, porém com uma diferença significativa das médias em relação à presença do trabalho na vida do idoso $(\mathrm{p}=0,046)$. Nele foi evidenciado que os idosos que não possuem trabalho apresentam piora na capacidade funcional comparados aos que trabalham ${ }^{(23)}$.

A dor é um dos principais fatores que limita a possibilidade da vivência do cotidiano pela pessoa idosa, impactando negativamente em sua QV e prejudicando a realização das atividades de vida diária, além da restrição da convivência social ${ }^{(26)}$.

Pesquisa evidencia que a presença da dor influencia negativamente a QV dos idosos ( $\mathrm{p}<$ $0,05)$, sendo necessário que os profissionais de saúde estejam atentos para identificar o quadro álgico, com o intuito de nortear e intervir de modo a minimizar este sofrimento ${ }^{(27)}$. Os impactos na QV gerados pela dor afetam a manutenção da autonomia $(\mathrm{p}=0,0004)$ da pessoa idosa, limitando o desempenho de suas atividades cotidianas, tornando-o dependente dentro de seu contexto social, econômico e cultural. Diante disso, a dor é um dos principais fatores que limitam a possibilidade do idoso sustentar seu cotidiano ${ }^{(27)}$.

Os domínios Funcionamento do Sensório (FS) e Morte e Morrer (MM) obtiveram as maiores médias (2,43 e 2,28 respectivamente), indicando melhor QV dos idosos. Esse dado corrobora com os valores médios encontrados em outros estudos (FS: $\mathrm{p}=0,000001$ a 0,$691 ; \mathrm{MM}: \mathrm{p}=0,02$ a 0,952 ), reforçando a influência positiva dos fatores inerentes a esses domínios na vida dos idosos ${ }^{(23,28-30)}$.

A pergunta que obteve maior escore no domínio Funcionamento do Sensório foi "Até que ponto o funcionamento dos seus sentidos (por exemplo, audição, visão, paladar, olfato e tato) afeta a sua capacidade de interagir com outras pessoas?". Estudos corroboram com esse resultado, afirmando que a capacidade funcional é um indicador de saúde na terceira idade, interferindo na saúde física e mental, independência na vida diária e integração social. Além disso, destacam o papel importante que as redes de apoio social exercem na velhice, sobretudo quando há necessidade de adaptação às perdas físicas e sociais ${ }^{(31)}$.

A QV da pessoa idosa envolve a manutenção de sua autonomia e capacidade funcional. Dessa maneira, o bom funcionamento sensorial na terceira idade permite a participação em atividades e a capacidade de interagir com outras pessoas, tornando o indivíduo independente de cuidados, resultando em melhoria de $\mathrm{QV}^{(14)}$.

A pergunta que obteve maior escore nas respostas do domínio Morte e Morrer foi "O quanto você tem medo de morrer?”. Vários estudos contemplam o mesmo resultado, e explicam que o confronto com a morte e a perda de parentes e amigos são comuns nessa fase da vida, retratando assim, a tendência de menor preocupação da pessoa idosa em relação à morte ${ }^{(28-30)}$.

A espiritualidade foi apontada como fator essencial para uma melhor aceitação da morte. Os autores afirmam que os idosos que possuem ligação com a religiosidade e a fé em seu cotidiano apresentam um melhor entendimento acerca do envelhecimento, compreendendo a morte como etapa natural do processo evolutivo, assim tendem a ter menores preocupações em relação à morte ${ }^{(23)}$.

Esse estudo tem como limitação o fato de ter sido realizado em uma população especifica e restrita a uma realidade local, portanto seus resultados não podem ser generalizados. Sugere-se a realização de estudos amplos para gerar evidencias nacionais e mundiais sobre a QV de idosos cardíacos, considerando o crescimento desse perfil populacional ao redor do mundo.

Espera-se que esse trabalho possa contribuir para o aperfeiçoamento e qualificação das boas práticas de profissionais de saúde e atendimento à essa população, incentivando e subsidiando ações, estratégias e programas de assistência voltados para essas questóes, especialmente as que mais comprometem a QV dos idosos.

\section{CONCLUSÃO}

O estudo evidenciou que a QV dos idosos com IC "precisa melhorar", ou seja, está aquém do ideal de ser boa ou ótima. O principal aspecto relacionado a melhor QV foi o bom funcionamento sensorial e a pouca preocupação com a morte. Já o pior aspecto que influencia na QV é a baixa renda e falta do lazer além da existência de dores físicas 
que limitam as atividades de vida diária. Há que considerar uma profunda reflexáo sobre o tema uma vez que é reconhecido o processo crescente de envelhecimento da população mundial e a existente oferta de recursos que possibilitam a garantia de melhores condiçóes de vida às pessoas.

Também, considerando os resultados desse estudo, é possível afirmar que os cuidados de

\section{REFERÊNCIAS}

1. Araújo AA, Sousa MM, Silva EP, Santos SR, Costa MML, Silva Filho IG. Perfil epidemiológico e clínico de pacientes acometidos por insuficiência cardíaca. Rev enferm UFPE [Internet]. 2014 Mar [citado 2018 mai 10]; 8(3): 509-13. Disponível em: 10.5205/r euol.5149-42141-1-SM.0803201403.

2. Comitê Coordenador da Diretriz de Insuficiência Cardíaca. Diretriz Brasileira de Insuficiência Cardíaca Crônica e Aguda. Arq Bras Cardiol [Internet]. 2018 [citado 2018 mai 13]; 111(3): 436-539. Disponível em: http://publicacoes. cardiol.br/portal/abc/portugues/2018/v11103/ pdf/11103021.pdf

3. Albuquerque DC, Neto JD, Bacal F, Rohde LEP, Bernardez-Pereira S, Berwanger O, et al. I Brazilian Registry of Heart Failure - Clinical Aspects, Care Quality and Hospitalization Outcomes. Arq Bras Cardiol [Internet]. 2015 Jun [citado 2018 mai 21]; 104(6): 433-42. Disponível em: https://doi. org/10.5935/abc.20150031.

4. Ferreira MCG, Tura LFR, Silva RC, Ferreira MA. Social representations of older adults regarding quality of life. Rev Bras Enferm [Internet]; 2017 [citado 2018 nov 19]; 70(4): 806-13. Disponível em: http://dx.doi.org/10.1590/0034-7167-20170097

5. Rodrigues GHP, Gebara OCE, Gerbi CCS, Pierri H, Wajngarten M. Depression as a Clinical Determinant of Dependence and Low Quality of Life in Elderly Patients with Cardiovascular Disease. Arq Bras Cardiol [Internet]. 2015 Jun [citado 2018 mai 10]; 104(6): 443-49. Disponível em: https:// www.scielo.br/pdf/abc/v104n6/pt_0066-782Xabc-20150034.pdf

6. Sawada NO, Paiva BSR, Paiva CE, Sonobe HM, García-Caro MP, Cruz-Quintana F. Transcultural approach to the health-related quality of life of cancer patients. Rev Rene [Internet]. 2017 enfermagem para com os idosos, especialmente os portadores de doenças crônicas, como a insuficiência cardíaca aqui abordada, devem ser refletidos e aperfeiçoados para além dos aspectos clínicos, levando em conta todos os domínios e aspectos que interferem na qualidade de vida, garantindo assim uma atenção integral e humanizada a saúde desses idosos.
Aug 21 [citado 2018 mai 10]; 18(3): 368-75. Disponível em: https://doi.org/10.15253/21756783.2017000300012

7. Ascef BO, Haddad JPA, Álvares J, Junior AAG, Costa EA, Acurcio FA, et al. Health-related quality of life of patients of Brazilian primary health care. Rev Saude Pública [Internet]. 2017 Sep 22 [citado 2018 mai 22]; 51(suppl 2): 1-12. Disponível em: https://doi.org/10.11606/S15188787.2017051007134

8. Pereira DS, Nogueira JAD, Silva CAB. Quality of life and the health status of elderly persons: a population-based study in the central sertão of Ceará. Rev Bras Geriatr Gerontol [Internet]. 2015 Dec [citado 2018 mai 22]; 18(4): 893-08. Disponível em: http://dx.doi.org/10.1590/18099823.2015.14123

9. Sousa CM, Farias RCP, Doula SM, Mafra SCT. Social representations about aging and social rights of the elderly: the case of programa municipal da terceira idade, Viçosa - Minas Gerais. Oikos: Revista Brasileira de Economia Doméstica [Internet]. 2016 Jun [citado 2018 jun 05]; 27(1): 135-56. Disponível em: https://www.periodicos. ufv.br/ojs/oikos/article/view/3721/1982

10. World Health Organization. Whoqol-old: Manual. WHO [Internet]. 2006 May [citado 2018 jun 08]. Disponível em: https://www.who.int/mental_ health/evidence/WHOQOL_OLD_Manual. pdf?ua $=1$

11. Santos PM. Principais Instrumentos de Avaliação da Qualidade de Vida de Idosos no Brasil: Vantagens e Desvantagens na Utilização. Corpoconsciência [Internet]. 2015 Aug [citado 2018 jun 08]; 19(2): 25-36. Disponível em: http://periodicoscientificos. ufmt.br/ojs/index.php/corpoconsciencia/article/ view/3948

12. World Health Organization. Whoqol-bref: Introduction, Administration, Scoring and Generic Version of the Assessment: Field Trial Version. 
WHO [Internet]. 1996 Dec [citado 2018 mai 25]. Disponível em: https://www.who.int/mental_ health/media/en/76.pdf

13. Fleck MPA, Louzada S, Xavier M, Chachamovich E, Vieira G, Santos L, et al. Aplicação da versão em português do instrumento abreviado de avaliação da qualidade de vida "WHOQOL-bref". Rev Saúde Pública [Internet]. 2000 Apr [citado 2018 jul 09]; 34(2): 178-183. Disponível em: https:// doi.org/10.1590/S0034-89102000000200012

14. Adamo CE, Esper MT, Bastos GCFC, Sousa IF, Almeida RJ. University of the Third Age: the impact of continuing education on the quality of life of the elderly. Rev bras geriatr geronto [Internet]. 2017 Aug [citado 2018 jul 12]; 20(4): 545-555. Disponível em: https://doi.org/10.1590/198122562017020.160192

15. BRASIL. Ministério da Saúde. Conselho Nacional de Saúde. Resolução n. 466, de 12 de dezembro de 2012. Aprova diretrizes e normas regulamentadoras de pesquisas envolvendo seres humanos. Brasília, Diário Oficial da Uniāo, 12 dez. 2012. Disponível em: https://bvsms.saude.gov.br/bvs/saudelegis/cns /2013/res0466_12_12_2012.html

16. Viana PAS, Neto JDC, Novais CT, Guimarães IF, Lopes YS, Reis BC. Profile of patients hospitalized to treat decompensated heart failure. SANARE [Internet]. 2018 Jun [citado 2018 out 28]; 17(1): 15-23. Disponível em: https://sanare.emnuvens. com.br/sanare/article/view/1218

17. Poffo MR, Assis AV, Fracasso M, Londero FOM, Alves SMM, Bald AP, et al. Profile of Patients Hospitalized for Heart Failure in Tertiary Care Hospital. Int J Cardiovasc Sci [Internet]. 2017 Jun [citado 2018 out 28]; 30(3): 189-98. Disponível em: https://www.scielo.br/pdf/ijcs/v30n3/23594802-ijcs-30-03-0189.pd

18. Kaufman R, Azevedo VMP, Xavier RMA, Geller M, Chaves RBM, Castier MB. Evolution of Heart Failure-related Hospital Admissions and Mortality Rates: a 12-Year Analysis. Int J Cardiovasc Sci [Internet]; 2015 Sep [citado 2018 out 29]; 28(4): 276-81. Disponível em: http://www.dx.doi.org/ $10.5935 / 2359-4802.20150040$

19. Areosa SVC. Providers elderly: the importance of resources to retirement the brazilian families. Barbarói [Internet]; 2015 Dec [citado 2018 out 30]; (45): 173-84. Disponível em: http://dx.doi. org/10.17058/barbaroi.v0i0.5527

20. Silva DMD, Vilela ABA, Nery AA, Duarte ACS, Alves MDR, Meira SS. Dynamics of intergenerational family relationships from the viewpoint of elderly residents in the city of Jequié (Bahia), Brazil. Ciênc saúde coletiva [Internet]; 2015 Jul [citado 2018 nov 04]; 20(7): 173-84.
Disponível em: https://doi.org/10.1590/1413-812 32015207.17972014

21. Ministéro do Planejamento, Desenvolvimento e Gestão. Pesquisa nacional por amostra de domicílios: síntese de indicadores 2015. Rio de Janeiro: IBGE [Internet]; 2016 [citado 2018 nov 04]. Disponível em: https://biblioteca.ibge.gov.br/ visualizacao/livros/liv98887.pdf

22. Rodrigues LR, Nader ID, Silva ATM, Tavares DMS, Assunção LM, Molina NPFM. Spirituality and religiosity related to socio-demographic data of the elderly population. Rev Rene [Internet]; 2017 Jul [citado 2018 nov 16];18(4): 429-36. Disponível em: https://doi.org/10.15253/21756783.2017000400002

23. Costa IP, Bezerra VP, Pontes MLF, Moreira MASP, Oliveira FB, Pimenta CJL, et al. Quality of life of elderly people and its association with work. Rev Gaúcha Enferm [Internet]; 2018 [citado 2018 jan 18]; 39. Disponível em: https://www.scielo.br/pdf/ rgenf/v39/en_1983-1447-rgenf-39-e2017-0213. pdf

24. Araújo, FF; Rocha, A. Meanings attached to sports in the elderly: note participant in musical encounters. PODIUM Sport, Leisure and Tourism Review [Internet]; 2016 May [citado 2018 nov 19]; 5(2): 38-55. Disponível em: https://doi. org/10.5585/podium.v5i2.157

25. Faria I, Ferreira VS, Dayrel JT. The art as art of existence: experiences of socialization of young people of origin popular in Salvador, Bahia. Reflexão e Ação [Internet]. 2018 May [citado 2018 nov 21]; 26(2): 130-148. Disponível em: https:// doi.org/ HYPERLINK "https://doi.org/10.17058/ rea.v26i2.11717”10.17058/rea.v26i2.11717

26. Carvalho AD, Souza EP. The Elderly and Chronic Pain: How to Live With Them. Mult Psic [Internet]; 2017 Nov [citado 2018 dez 08]; 11(38): 689-700. Disponível em: http://idonline.emnuvens.com.br/ id

27. Valero MC, Faria MQG, Lucca PSR. Evaluation and treatment of chronic pain in elderly patient. Revista Thêma et Scientia [Internet]; 2015 Dec [citado $2018 \mathrm{dez}$ 07]; 5(2): 129-38. Disponível em: https://www.fag.edu.br/upload/ arquivo/1457726942.pdf

28. Andrade JF, Brito MFSF, Souza LPF, Guimarães ALS, D'Angelo MFSV, Silva CSO. Quality of life of elderly in a reference center in Minas Gerais, Brazil. Rev Med UFC [Internet]; 2018 [citado 2018 jan 10]; 58(1): 26-30. Disponível em: https:// doi.org/10.20513/2447-6595.2018v58n1p26-30

29. Ermel RC, Caramelo AC, Fracolli LA, Ortiz FVBC, Zutin TLM, Gianini SHS, et al. Perception on the life quality of Portuguese and Brazilian 
elderly. REAS [Internet]; 2017 Nov [citado 2018 jan 10]; 9(2): 1315-1320. Disponível em: https:// doi.org/10.25248/REAS98_2017

30. Esteves M, Vendramini SHF, Santos MLSG, Brandáo VZ, Soler ZASG, Lourenção LG. Quality of life of hypertensive and diabetic elderly in an outpatient clinic. Medicina [Internet]; 2017 [citado 2018 jan 13]; 50(1): 18-28. Disponível em: http://dx.doi.org/10.11606/issn.2176-7262. v50i1p18-28

31. Possatto JM, Rabelo DF. Psychological health, functional capacity and social support in the elderly. Revista Kairós: Gerontologia [Internet]; 2017 [citado 2018 jan 03]; 20(2): 45-58. Disponível em: https://doi.org/10.23925/2176-901X. 2017v20i2p45-5 